\title{
EXTRAVERSION, NEUROTICISM AND SUSCEPTIBILITY TO POSITIVE AND NEGATIVE MOOD INDUCTION PROCEDURES
}

\author{
RANDY J. LARSEN and Timothy KeTELAaR \\ Department of Psychology, University of Michigan, Ann Arbor, MI 48I09, U.S.A.
}

(Received 23 January 1989)

\begin{abstract}
Summary-Recent correlational research suggests that Extraversion is associated with a predisposition to experience positive affect, whereas Neuroticism is associated with a predisposition to experience negative affect. Using Gray's (A Model for Personality, pp. 246-276, 1981) terms, such results may be due to differential sensitivity to signals of reward and punishment on the part of Extraverts and Neurotics, respectively. Assuming that signals of reward generate positive affect and signals of punishment (or frustrative non-reward) generate negative affect, we hypothesized that the efficacy of a negative affect induction would be better predicted from Neuroticism than Extraversion scores, whereas the efficacy of a positive affect induction should be better predicted from Extraversion than Neuroticism scores. In the current study a laboratory mood induction technique (false feedback of success and failure) was used to induce positive and negative affect, and its effectiveness was assessed using standard mood adjective ratings. Results support the hypothesis that Extraverts (compared to Introverts) show heightened emotional reactivity to positive (but not negative) mood induction procedures, whereas Neurotics (compared to Stable individuals) show heightened emotional reactivity to negative (but not positive) mood induction procedures. Results are discussed in terms of an emotion-based approach to personality theory, and directions for future research are suggested.
\end{abstract}

Aspects of personality can be viewed as affect-dispositional dimensions. This statement assumes, at a minimum, that relationships can be found between measures of personality and measures of typical affective states (Tellegen, 1985). Several studies provide evidence for such relationships. For example, Costa and McCrae (1980) found that Extraversion traits correlated strongly with concurrent positive affect, and Neuroticism traits correlated strongly with concurrent negative affect. These same relationships were also found in a 10-yr follow-up study reported by Costa and McCrae (1980). These authors conclude that "Extraversion ... predisposes individuals toward positive affect, whereas Neuroticism predisposes individuals toward negative affect" (p. 673). Similar conclusions have been drawn by other researchers (e.g. Emmons and Diener, 1985; O'Malley and Gillette, 1984; Warr, Barter and Brownbridge, 1983; Watson, 1988).

Such conclusions could only hold if positive and negative affect are at least somewhat orthogonal to each other, an assumption that is supported by recent research (Watson and Tellegen, 1985; Watson, Clark and Tellegen, 1988). Positive and negative affect emerge as separate dimensions accounting for the majority of variance in factor analyses of cross-sectional as well as longitudinal studies of emotion (Clark and Watson, 1988; Zevon and Tellegen, 1982). The recent revision of the Mood Affect Adjective Checklist (MAACL; Zuckerman and Lubin, 1985) now provides separate positive and negative affect scale scores. It appears that positive and negative affect, especially when assessed over longer time periods (Diener and Emmons, 1984), are orthogonal dimensions. As such, positive and negative affect can correlate differentially (not simply with opposite sign) with external variables such as personality, social activities, and physical symptoms (Watson, 1988; Watson and Tellegen, 1985). In the domain of emotion, positive and negative affect thus emerge as the 'Big Two' affective dimensions (Tellegen, 1985).

In the personality domain two major dimensions also emerge with enough potency and regularity to be similarly considered as the 'Big Two' (Eysenck, 1967; Wiggins, 1968) personality dimensions: Extraverison and Neuroticism. Certainly many other primary personality dimensions exist (e.g. McCrae and Costa, 1987), but higher order conceptions of personality agree that Extraversion and Neuroticism define major axes of the individual difference space. These two personality dimensions also appear to relate more strongly and more consistently to affect than other personality dimensions (Costa and McCrae, 1980). 
The merging of personality and affective dimensions is highlighted by Tellegen's (1985) recent work. Tellegen (1985) views the relationship between personality and mood to be so overlapping that he terms two of the most powerful second-order dimensions to emerge from his program of personality scale construction as Positive and Negative Emotionality. Positive Emotionality consists of Extraversion/sociability traits that appear to promote positive emotional experiences. Negative Emotionality is associated with a cluster of primary Neurotic traits, such as stress reaction, alienation, and self-descriptions of worry, anxiety, feeling victimized and resentfulness. Such a trait cluster appears to foster negative emotional experiences. Positive and Negative Emotionality are affect-relevant personality traits that in turn relate to traditional inventory measures of Extraversion and Neuroticism respectively (e.g. Cattell's 16 PF, Eysenck's EPI, Guilford and Zimmerman's Temperament Schedule).

Beyond a descriptive account of personality in affect-dispositional terms, an important question remains as to why Extraversion should be related to positive affect and why Neuroticism should be related to negative affect? A step toward a theoretical account of these individual differences in emotional functioning can be deduced from Gray's theory of personality functioning $(1970,1981$, 1987; see also Fowles, 1980, 1987 for discussions of Gray's theoretical work relevant to mood and personality). Based on neurophysiological research (mostly with animal models) Gray proposes the existence of two emotion-based neuronal systems responsible for behavioral regulation: the behavioral activation system (BAS) is thought to control behavior in the presence of signals of reward, and the behavioral inhibition system (BIS) is thought to regulate behavior in response to signals of punishment or frustrative non-reward. These two systems are thought to be orthogonal and each gives rise to a major personality dimension: sensitivity to signals of reward is linked to Extraversion traits, and sensitivity to punishment is linked to Neuroticism traits. Individuals are thought to differ from each other in the relative strength of these two signal-sensitivity systems, such that these systems are often conceptualized in trait-like terms (Gorenstein and Newman, 1980; Newman, 1987; Newman, Widom and Nathan, 1985; Zuckerman, 1987) or as having behavioral consequences that represent trait dimensions (Fowles, 1980, 1987; Zuckerman, 1987).

For our purposes, it is important to suggest the possibility that positive and negative affect are the subjectively reportable representations of reward-signal sensitivity and punishment-signal sensitivity, respectively. Sensitivity to reward is an appetitive motivational system that appears to activate behavior through positive affect. Sensitivity to punishment is an aversive motivatioinal system that appears to inhibit behavior through negative affect. When confronted with reward signals one experiences positive affect, and when confronted with punishment signals one experiences negative affect. It seems likely, then, that individual differences in susceptibility to positive and negative affect should be related to the personality dimensions associated with sensitivity to signals of reward and punishment, respectively.

Gray's work is actually a reformulation and extension of Eysenck's (e.g. Eysenck, 1967) prior work on Extraversion and Neuroticism. Eysenck (1987) points out that, in factor analyses of personality scales, measures thought to assess the BIS tend to align near the Neuroticism dimension, whereas measures thought to assess the BAS tend to correlate with Extraversion scores (as well as with Psychoticism scores). In this study we will therefore use the unrotated dimensions of Extraversion and Neuroticism. More important for our rationale, Eysenck has long held that personality predisposes individuals to specific forms of affect, mainly due to differential biological thresholds for specific emotional and motivational states. In particular, Extraverts are likely to experience positive affect more frequently than Introverts. Neurotic individuals, on the other hand, are likely to experience negative affect more frquently than Stable individuals (cf. Eysenck and Eysenck, 1985, p. 141). More recently, Eysenck (1987) suggested that, since positive affect and negative affect are best conceptualized as separate dimensions, it seems reasonable to hypothesize that Extraversion represents susceptibility to positive affect and Neuroticism represents susceptibility to negative affect. M. Eysenck (1987) cites Gray's theory as a rationale for this hypothesis, implying that both H. J. Eysenck's and Gray's approaches make similar predictions in terms of personality and susceptibility to affective states.

In a recent theoretical paper, Strelau (1987) also hypothesized that, since Extraverts are more sensitive to signals of reward, and since signals of reward are the source of positive affect, Extraverts should be more susceptible to positive affect than Introverts. Similarly, since Neuroticism is defined 
as sensitivity to signals of punishment, and since signals of punishment are the source of negative affect, Neurotics should therefore be more susceptible to negative affect than Stable or non-Neurotic individuals. This hypothesis, if true, would account for the correlational findings that Extraverts report more positive affect (but about the same amount of negative affect) than Introverts, and Neurotics report more negative affect (but about the same amount of positive affect) than Stable $S$ s. Moreover, this hypothesis of differential susceptibility to positive and negative affect goes further than postulating a simple correlation between personality dimensions and certain affective states. The theories discussed above imply that proneness to experience differential levels of positive or negative affect depends on individual differences in specific brain structures. These individual differences in brain structures are the hypothesized underlying cause of both the affective states (positive and negative) and the personality dimensions (Extraversion and Neuroticism). Consequently, if this hypothesis is true, Extraverts should be more responsive than Introverts to positive affect inductions, whereas Neurotics should be more responsive than Stable individuals to negative affect inductions. In other words, Extraverts and Introverts should show differential sensitivity to positive but not negative affect, whereas Neurotics and Stable individuals should show differential sensitivity to negative but not positive affect.

The present study was designed to test this hypothesis using an experimental rather than a correlational design. We used a standard laboratory mood induction procedure to manipulate either positive or negative affect. Susceptibility to the induced emotion was assessed using standard mood adjective rating tasks. If the hypotheses regarding differential susceptibility to affective states are correct then we should find that Extraversion is associated with a sensitivity to positive (but not negative) affect inductions and Neuroticism is associated with a sensitivity to negative (but not positive) affect inductions. In other words, the strength of our negative affect induction should be better predicted from Neuroticism scores than Extraversion scores, whereas the strength of our positive affect inductions should be better predicted from the Extraversion scores than the Neuroticism scores. Putting this hypothesis in still another way, negative affect inductions should 'take' better for Neurotic than Stable individuals, whereas positive affect inductions should 'take' better for Extraverted than Introverted individuals.

\section{METHOD}

\section{Subjects}

The $S$ s in this study were 67 college undergraduates who participated as part of their introductory psychology course. Participants came to the laboratory in groups of 4-6 for this study.

\section{Measures}

After arriving in the laboratory, $S$ s were seated at a table and asked to complete the Eysenck Personality Inventory (EPI; Eysenck and Eysenck, 1964). This is a 57-item true-false questionnaire that has been extensively validated and widely used in personality research. This questionnaire yields Extraversion, Neuroticism, and Lie scores.

\section{Mood induction procedure}

The mood induction procedure used in this study was a form of the false feedback technique (e.g. Notarius, Wemple, Ingraham, Burns and Kollar, 1982). After completing the EPI, participatnts were told that the next task in the study was part of a large-scale norming project for a recently developed intelligence test. This test was described as a measure of a newly discovered form of intelligence (Syncretic Skill), and that we were part of a nation-wide norming study. It was stressed to the $S \mathbf{S}$ that they should do their best in order to ensure a representative assessment of their Syncretic Skill ability. The measure was referred to as the Test of Syncretic Skill (or TOSS). The ability that this test measures was described to the $S \mathrm{~s}$ as:

\footnotetext{
"Syncretic Skill refers to a specific mental ability. This ability is important for a number of reasons. It refers to the capacity of a person's brain to quickly and efficiently process abstract information. This is a much different ability than the simple 'book knowledge' measured by most intelligence tests. Nevertheless, Syncretic Skill, or the ability to quickly perceive and process abstract relations, is an important part of overall intelligence. The Test of Syncretic Skill (TOSS) measures this ability to process complex information efficiently".
} 
In reality, the TOSS consisted of several subtests adapted from Thurstone's (1938) book on primary mental abilities. For example, one section of the TOSS consisted of maze tracing puzzles, another section consisted of block diagrams where the $S$ s had to estimate the number of blocks in the diagram, and another section consisted of visual analogies. Each section of the TOSS was timed during administration in such a way that most $S$ s were unable to complete all the exercises in each section within the time limit.

Upon completion of the TOSS, the experimenter gave the $S$ s a packet of personality questionnaires to complete while he went to another room to ostensibly score their TOSS measures. In reality, scoring and feedback sheets for the TOSS had been prepared ahead of time, and the experimenter simply transferred the $S$ s identification number and date onto one of the feedback sheets. After approx. 10 min the experimenter returned and suggested that, since most $S$ s tested so far were curious about how they performed on the TOSS, it might be interesting for the $S$ s to go over their own scores. The experimenter then gave each $S$ their own feedback form indicating how they, the $S$, had performed on the TOSS.

There were two forms of feedback sheets. One form (the success condition) indicated that the $S$ had performed a good deal above average on the TOSS (in the upper third), and that they clearly had superior Syncretic Skill ability. The other feedback form (the failure condition) indicated that the $S$ had performed quite a bit below average on the TOSS (in the lower third), and that they were clearly deficient in their Syncretic Skill ability. A narrative description of what it means to have a high or low score on the TOSS was also included on the feedback sheets to reinforce the $S s^{\prime}$ understandings of the meaning of their own scores.

The assignment of $S \mathrm{~s}$ to the failure or success conditions was determined randomly. Thirty-seven $S$ s received failure feedback and $30 S$ s received success feedback. The provision of success and failure feedback on tasks in which the $S$ s are ego-involved has been widely used to induce positive and negative affect respectively (e.g. Polivy, 1981; Salovey and Rodin, 1984).

\section{Dependent measures}

The dependent measure in this study consisted of a mood adjective rating task. $S$ s were asked to "Please rate how you are feeling right now" by placing a slash-mark on a line separating two opposing mood adjectives. The mood-adjectives were taken from Mackay (1980). Examples of the bipolar mood adjectives used in this study are: Happy-Sad, Clear-Dull, Comfortable-Uncomfortable, and Tense-Relaxed. The line separating the bipolar adjectives was divided into 31 equal segments, and each item was scored by assigning an ordinal number to the segment through which the $S$ placed his or her slash. Item responses were aggregated to form a single total score (ranging from +15 to -15 ) indicating how positive or negative the subject reportedly felt, with a more positive number indicating a more positive mood. Mood adjective ratings such as these are widely used in the assessment of the extent to which a laboratory mood induction task is effective (Mackay, 1980).

\section{RESULTS}

The major hypothesis under investigation is that the strength or effectiveness of the positive affect induction should be better predicted from Extraversion scores than Neuroticism scores, whereas the strength or effectiveness of the negative affect induction should be better predicted from Neuroticism scores than Extraversion scores. Correlations were computed between the personality variables (Extraversion and Neuroticism) and the self-report of mood after the mood induction procedures. These correlations were done separately for the positive and negative affect induction conditions, and are presented in Table 1.

The correlations in Table 1 indicate that Neuroticism shows a strong relationship to the affect induction effect only when negative affect was manipulated. Extraversion, on the other hand, shows a strong relationship to the affect induction effect only when positive affect was manipulated. In other words, the effectiveness of the positive and negative affect inductions differed depending on personalities of the $S$ s; Extraversion appears to be associated with the effectiveness of the positive affect induction but not the negative affect induction, Neuroticism appears to be associated with 
Table 1. Correlations between personality scores and mood within conditions

\begin{tabular}{lccccc}
\hline & \multicolumn{2}{c}{$\begin{array}{c}\text { Negative affect } \\
\text { condition }\end{array}$} & & \multicolumn{2}{c}{$\begin{array}{c}\text { Positive affect } \\
\text { condition }\end{array}$} \\
\cline { 2 - 3 } & $\begin{array}{c}\text { Neuroticism } \\
(N=37)\end{array}$ & $\begin{array}{c}\text { Extraversion } \\
(N=37)\end{array}$ & & $\begin{array}{c}\text { Neuroticism } \\
(N=30)\end{array}$ & $\begin{array}{c}\text { Extraversion } \\
(N=30)\end{array}$ \\
\hline Mood measuret & $-0.30^{*}$ & -0.03 & & 0.01 & $0.25^{*}$ \\
\hline $\begin{array}{l}* \\
P\end{array}<0.05$. \\
+ High scores on the mood measure reflect more positive reported affect.
\end{tabular}

the effectiveness of the negative affect induction but not the positive affect induction. This suggests that if one wanted to predict the effects of a mood induction procedure from personality scores, one would be better able to predict the efficacy of a negative affect induction from Neuroticism rather than Extraversion scores, whereas the efficacy of a positive affect induction would be better predicted from Extraversion rather than Neuroticism scores. This lends some support to the hypothesis that positive affect inductions 'take' better for Extraverts than Neurotics, whereas negative affect inductions 'take' better for Neurotics than Extraverts.

Another way to examine the question of differential susceptibility to affective states is to look at differences in mean levels of mood between different personality groups. Extreme groups were created on both the Extraversion and Neuroticism scales by dividing the sample at the median on these two dimensions. That is, 'Introverted' and 'Extraverted' groups were created by splitting the sample at the median on the Extraversion score. Similarly, 'Stable' and 'Neurotic' groups were created by splitting the same sample at the median on the Neuroticism score. Because Extraversion and Neuroticism were correlated at 0.09 in this sample it seems appropriate to use the medians to create extreme groups. In the comparisons that follow, we compared the groups created by splitting the sample on the Extraversion scale, and then compared the groups created by splitting the same sample on the Neuroticism scale.

Group means within the mood induction conditions are presented in Table 2. An ANOVA was conducted on the factors of Extravert vs Introvert and positive vs negative mood induction condition. The main effect for the Extravert/Introvert factor was significant $(F=3.85, P<0.05)$, while the main effect for mood induction condition was not significant $(F=2.15, P<0.15)$. The interaction term was marginally significant $(F=3.28, P<0.10)$. A similar ANOVA was conducted using the Neurotic vs Stable and the positive vs negative induction factors. Again, the personality main effect was significant $(F=8.20, P<0.01)$, while neither the main effect for mood nor the interaction term were significant.

To better portray these group differences, the means on the mood scores within each condition are plotted separately for the four personality groups in Fig. 1. It can be seen that the difference between the Introvert and Extravert groups is greatest in the positive affect induction condition. The difference between the Stable and Neurotic groups, on the other hand, is greatest in the negative affect induction condition.

One unusual finding is that the mood of the Stable group in the negative affect condition was actually slightly more positive than the mood of the Stable group that underwent the positive mood induction. We would expect the moods of all the groups to be more negative in the negative than the positive induction condition. The finding that the mood of the Stable groups remained fairly consistent across the positive and negative induction conditions suggests that these individuals might somehow be engaging in a form of 'mood repair' (Isen, 1984) or utilizing a mood regulation strategy (Morris and Reilly, 1987) during the course of the experiment. The fact that the failure feedback had no appreciable influence on the moods of the Stable individuals suggests that these persons are truly 'stable' in the sense of maintaining their mood states across rather extreme

Table 2. Means for personality groups within conditions

\begin{tabular}{cccc}
\hline \multicolumn{2}{c}{$\begin{array}{c}\text { Negative affect } \\
\text { condition }\end{array}$} & \multicolumn{2}{c}{$\begin{array}{c}\text { Positive affect } \\
\text { condition }\end{array}$} \\
\hline Stable & Neurotic & Stable & Neurotic \\
11.28 & -3.00 & 9.59 & -2.34 \\
Introvert & Extravert & Introvert & Extravert \\
-0.88 & 1.79 & 0.29 & 13.53 \\
\hline
\end{tabular}




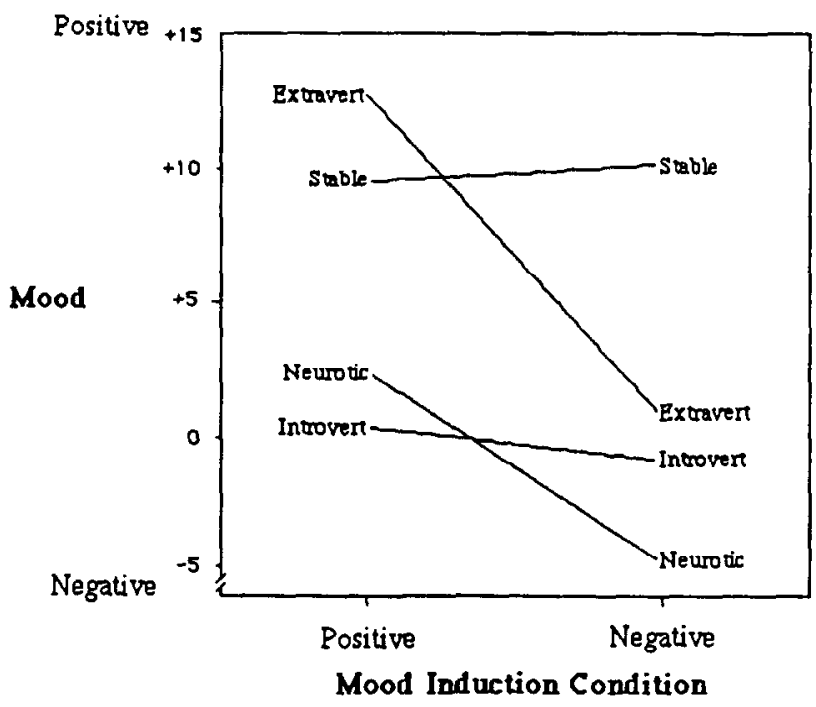

Fig. 1. Mean mood score as a function of personality group and affect induction condition.

conditions. All other groups, however, showed the appropriate directional differences in mood across conditions.

This brings us to the question of the overall main effect for the mood induction procedures. When we averaged across all persons and compared the positive affect induction condition to the negative affect induction condition, only a marginally significant difference was found. The mean mood score in the positive affect condition was 6.16 , and the mean mood score in the negative affect induction condition was $1.36(t=-1.31, P<0.10)$. This suggests that, over all $S \mathrm{~s}$, the affect induction procedures were rather weak and did not result in a conventionally significant main effect. However, as the above personality results demonstrate, there was an interaction between the type of mood induced and the personality characteristics of the $S \mathrm{~s}$. This interaction suggests that the negative affect induction effect was more strongly associated with the Neuroticism than the Extraversion dimension, whereas the positive affect induction effect was more strongly associated with the Extraversion than the Neuroticism dimension.

\section{DISCUSSION}

The present study sought to address the question of why Extraversion is found to correlate with characteristic level of positive affect and Neuroticism is found to correlate with characteristic level of negative affect (e.g. Costa and $\mathrm{McCrae}, 1980$ ). Such correlational research usually asks $S$ s how they typically feel emotionally, and the results suggest that the Extraverted $S$ s typically feel more positive affect (but about the same amount of negative affect) than Introverts, whereas the Neurotic $S$ s typically feel more negative affect (but about the same amount of positive affect) than Stable $S$ s. The present study pushes this logic further and hypothesizes that, given the appropriate emotion-provoking circumstances, Extraverts are more likely than Introverts to respond with positive affect, whereas Neurotics are more likely than Stables to respond with negative affect. In other words, we hypothesized that Extraversion is associated with a higher probability of experiencing positive affective states and Neuroticism is associated with a higher probability of experiencing negative affective states during an in vivo mood-induction task.

This hypothesis draws on Gray's (1981) approach to personality functioning, but uses the Eysenckian dimensions of Extraversion and Neuroticism as indicators of sensitivity to signals of reward and sensitivity to signals of punishment, respectively. It is a small theoretical leap to assume that sensitivity to signals of reward is experientially manifest as positive emotional reactivity, whereas sensitivity to signals of punishment is experientially manifest as negative emotional reactivity. This assumption has been made by other researchers (e.g. Eysenck, 1987; Strelau, 1987), but never empirically tested. The hypotheses that follow from this assumption are (1) that Extraverts (compared to Introverts) should show heightened reactivity to positive (but not 
negative) mood induction procedures and (2) that Neurotics (compared to Stable individuals) should show heightened reactivity to negative (but not positive) mood induction procedures.

In the present study, we tested this hypothesis by examining the differential effectiveness of positive and negative mood induction procedures. We found that Introverts and Extraverts differed significantly from each other in their emotional response to the positive but not the negative mood induction. Neurotic and Stable individuals differed from each other significantly only in their emotional response to the negative but not the positive mood induction. In other words, we were better able to predict the efficacy of the positive mood induction from Extraversion rather than Neuroticism scores, whereas the efficacy of the negative mood induction was better predicted from Neuroticism scores rather than the Extraversion scores.

Because the two behavioral patterns of Extraversion and Neuroticism are thought to be based on biological structures intimately involved with the experience of affect (Eysenck, 1967), we suggest that these two personality dimensions also represent specific vulnerabilities to particular affective states. That is, different persons are differentially 'prepared' to respond with specific emotions given the same stimulus conditions. Extraversion appears to be associated with a preparedness to respond with stronger positive than negative affect under the appropriate circumstances, whereas Neuroticism appears to be associated with a preparedness to respond with stronger negative than positive affect under the appropriate circumstances. Such results, obtained in the present study using laboratory mood induction procedures, can account for the correlational findings that Extraverts experience more positive affect in their lives over time, whereas Neurotics experience more negative affect in their lives over time. The current results also provide some experimental support for Costa and McCrae's (1980) statement that "Extraverted traits contribute to one's positive enjoyment ..., although they do not generally appear to reduce the unpleasantness of adverse circumstances. Neurotic traits predispose one to suffer more accutely from one's misfortunes, but they do not necessarily diminish one's joy or pleasures" (p. 674).

There are certain theoretical advantages to be gained from research relating personality traits to emotion concepts. First, such efforts make it clear that certain personality traits may be regarded as mood-dispositional dimensions. This helps us understand that characteristic ways of emotional responding provide a degree of affective organization to personality processes. Secondly, such efforts help us become more precise about the nature and function of the personality traits we study. That is, our understanding of different personality constructs grows as we discover how emotions are involved in the behaviors and experiences that define various personality dimensions. And thirdly, such efforts help us understand the nature of the emotional processes we study. For example, the nature of the relationship between positive and negative affect is a topic of much current theoretical interest (e.g. Clark and Watson, 1988). When, in the current study, we examined positive and negative affect in relation to two distinct personality systems, we provide a way of thinking about the distinction between positive and negative affect. Thus research on the relations between personality and emotion could potentially benefit several areas of psychological inquiry.

Based on the results of the current study, future research might profitably examine the generality of differential susceptibility to positive and negative mood inductions. For example, different mood induction procedures might be used to make sure that Extraverts and Introverts are not just differentially susceptible to the emotional impact of success and failure feedback. The Velten mood induction procedure (Velten, 1968) might be tried, or other mood induction procedures such as imagery, films, music or hypnosis could be evaluated. Other methods for assessing the effectiveness of mood induction procedures might also be examined. For example, will Extraversion and Neuroticism scores differentially predict facial responsiveness to positive and negative affect inductions? The assumption that certain personality traits predispose individuals to experience specific emotions leads to a variety of interesting and important questions about the nature and consequences of known individual differences. 


\section{REFERENCES}

Clark L. A. and Watson D. (1988) Mood and the mundane: relations between daily life events and self-reported mood. J. Person. Soc. Psychol. 54, 296-308.

Costa P. T. and McCrae R. R. (1980) Influence of Extraversion and Neuroticism on subjective well-being: happy and unhappy people. J. Person. Soc. Psychol. 38, 668-678.

Diener E. and Emmons R. A. (1984) The independence of positive and negative affect. J. Person. Soc. Psychol. 47, $1105-1117$.

Emmons R. A. and Diener E. (1985) Personality correlates of subjective well-being. Person. Soc. Psychol. Bull. 11, 89-97.

Eysenck H. J. (1967) The Biological Basis of Personality. Thomas, Springfield, Ill.

Eysenck H. J. and Eysenck M. (1985) Personality and Individual Differences: A Natural Science Approach. Plenum Press. New York.

Eysenck H. J. and Eysenck S. B. G. (1964) Manual of the Eysenck Personality Inventory. Educational and Industrial Testing Service, San Diego, Calif.

Eysenck M. (1987) Trait theories of anxiety. In Personality Dimensions and Arousal (Edited by Strelau J. and Eysenck H. J.), pp. 79-97. Plenum Press, New York.

Fowles D. (1980) The three arousal model: implications of Gray's two-factor learning theory for heart rate, electrodermal activity, and psychopathology. Psychophysiology 17, 87-104.

Fowles D. (1987) Application of a behavioral theory of motivation to the concepts of anxiety and impulsivity. J. Res. Person. 21, 417-435.

Gorenstein E. E. and Newman J. P. (1980) Disinhibitory psychopathology: a new perspective and a model for research. Psychol. Rev. 87, 301-315.

Gray J. A. (1970) The psychophysiological basis of introversion-extraversion. Behav. Res. Ther. 8, $249-266$.

Gray J. A. (1981) A critique of Eysenck's theory of personality. In A Model for Personality (Edited by Eysenck H. J.), pp. 246-276. Springer, New York.

Gray J. A. (1987) Perspectives on anxiety and impulsivity: a commentary. J. Res. Person. 21, 493-509.

Isen A. M. (1984) Toward understanding the role of affect in cognition. In Handbook of Social Cognition (Edited by Wyer R. and Srull T.), Vol. 3, pp. 179-236. Erlbaum, Hillsdale, N.J.

McCrae R. R. and Costa P. T. (1987) Validation of the five-factor model of personality across instruments and observer. J. Person. Soc. Psychol. 52, 81-90.

Mackay C. J. (1980) The measurement of mood and psychophysiological activity using self-report techniques. In Techniques in Psychophysiology (Edited by Martin 1. and Venables P.), pp. 501-562. Wiley, New York.

Morris W. N. and Reilly N. P. (1987) Toward the self-regulation of mood: theory and research. Motiv. Emot. 11, 21 S-249.

Newman J. P. (1987) Reactions to punishment in extraverts and psychopaths: implications for the impulsive behavior of disinhibited individuals. J. Res. Person. 21, 464-480.

Newman J. P., Widom C. S. and Nathan S. (1985) Passive-avoidance in syndromes of disinhibition: psychopathy and extraversion. J. Person. Soc. Psychol. 48, 1316-1327.

Notarius C. I., Wemple C., Ingraham L. J., Burns T. J. and Kollar E. (1982) Multichannel responses to an interpersonal stressor: interrelationships among facial display, heart rate, self-report of emotion, and threat appraisal. J. Person. Soc. Psychol. 43, 400-408.

O'Malley M. N. and Gillette C. S. (1984) Exploring the relations between traits and emotions. J. Person. 52, $274-284$.

Polivy J. (1981) On the induction of emotion in the laboratory: discrete moods or multiple affect states. J. Person. Soc. Psychol. 41, 803-817.

Salovey P. and Rodin J. (1984) Some antecedents and consequences of social-comparison jealousy. J. Person. Soc. Psychol. 47, 780-792.

Strelau J. (1987) Emotion as a key concept in temperament research. J. Res. Person. 21, 510-528.

Tellegen A. (1985) Structures of mood and personality and their relevance to assessing anxiety, with an emphasis on self-report. In Anxiety and the Anxiety Disorders (Edited by Tuma A. H. and Mason J.), pp. 681-706. Erlbaum, Hillsdale, N.J.

Thurstone L. L. (1938) Vectors of the Mind: Multiple-Factor Analysis for the Isolation of Primary Traits. University of Chicago Press, Chicago.

Velten E. (1968) A laboratory task for the induction of mood states. Behav. Res. Ther. 6, 473-482.

Warr P., Barter J. and Brownbridge G. (1983) On the independence of positive and negative affect. J. Person. Soc. Psychol. 44, 644-651.

Watson D. (1988) Intraindividual and interindividual analyses of positive and negative affect: their relation to health complaints, perceived stress, and daily activities. J. Person. Soc. Psychol. 54, 1020-1030.

Watson D. and Tellegen A. (1985) Toward a consensual structure of mood. Psychol. Bull. 98, 219-235.

Watson D., Clark L. A. and Tellegen A. (1988) Development and validation of brief measures of Positive and Negative Affect: the PANAS Scales. J. Person. Soc. Psychol. 54, 1063-1070.

Wiggins J. S. (1968) Personality structure. Annual Review of Psychology, Vol. 19. Annual Reviews, Palo Alto, Calif.

Zevon M. A. and Tellegen A. (1982) The structure of mood change: an idiographic/nomothetic anaiysis. J. Person. Soc. Psychol. 43, 111-122.

Zuckerman M. (1987) A critical look at three arousal constructs in personality theories: optimal levels of arousal, strength of the nervous system, and sensitivities to signals of reward and punishment. In Personality Dimensions and Arousal (Edited by Strelau J. and Eysenck H. J.), pp. 217-232. Plenum Press, New York.

Zuckerman M. and Lubin B. (1985) Manual for the Multiple Affect Adjective Checklist-Revised. Educational and Industrial Testing Service, San Diego, Calif. 